\title{
Stochastic Dynamics of Electrical Membrane with Voltage-Dependent Ion Channel Fluctuations
}

\author{
Hong QIAN ${ }^{1}$, Xue-Juan Zhang ${ }^{2}$ and Min QIAN ${ }^{3}$ \\ 1 Department of Applied Mathematics, University of Washington, Seattle, WA 98195-3925, \\ USA; College of Mathematics, Jilin University, Changchun, Jilin 130012, PRC \\ 2 Department of Mathematics, Zhejiang Normal University, Jinhua, Zhejiang 321004, PRC \\ 3 School of Mathematical Sciences, Peking University, Beijing 100871, PRC
}

PACS 05.40.Ca - fluctuation phenomena

PACS 05.70.Ln - statistical thermodynamics

PACS 87.16.dp - transport processes

\begin{abstract}
A}$ Brownian ratchet like stochastic theory for the electrochemical membrane system of Hodgkin-Huxley (HH) is developed. The system is characterized by a continuous variable $Q_{m}(t)$, representing mobile membrane charge density, and a discrete variable $K_{t}$ representing ion channel conformational dynamics. A Nernst-Planck-Nyquist-Johnson type equilibrium is obtained when multiple conducting ions have a common reversal potential. Detailed balance yields a previously unknown relation between the channel switching rates and membrane capacitance, bypassing Eyring-type explicit treatment of gating charge kinetics. From a molecular structural standpoint, membrane charge $Q_{m}$ is a more natural dynamic variable than potential $V_{m}$; our formalism treats $Q_{m}$-dependent conformational transition rates $\lambda_{i j}$ as intrinsic parameters. Therefore in principle, $\lambda_{i j}$ vs. $V_{m}$ is experimental protocol dependent, e.g., different from voltage or charge clamping measurements. For constant membrane capacitance per unit area $C_{m}$ and neglecting membrane potential induced by gating charges, $V_{m}=Q_{m} / C_{m}$, and HH's formalism is recovered. The presence of two types of ions, with different channels and reversal potentials, gives rise to a nonequilibrium steady state with positive entropy production $e_{p}$. For rapidly fluctuating channels, an expression for $e_{p}$ is obtained.
\end{abstract}

Introduction. - Hodgkin-Huxley's $1952(\mathrm{HH})$ theory of neuronal electrical impulse generation [1, in terms of voltage-dependent $\mathrm{Na}^{+}$and $\mathrm{K}^{+}$ion channels embedded in cell membrane that is treated as a leaky capacitor, remains one of the greatest biophysical understandings of living processes 2 . Following the fundamental observations that individual ion channels stochastically fluctuate within discrete conducting states 3,4 and recent laboratory experiments on voltage-dependent channel conformational changes [5 7], the $\mathrm{HH}$ theory has been extended to a mesoscopic scale [8,9]. While the literature on stochastic HH dynamics is rapidly growing [10,11, none has provided it a sound statistical physics formulation including equilibrium electrochemical fluctuations as one of its appropriate limits. One notices that action potentials are highly nonlinear and far from nonequilibrium phenonmenon driven by sustained $\mathrm{Na}^{+}$and $\mathrm{K}^{+}$ion concentration differences across excitable, living cell membrane 1, 2, 12. However, when such a driving force is absent, a passive lipid bilayer with fluctuating ion channels should observe Nernst-Planck (NP) equilibrium and obey Nyquist-Johnson's (NJ) charge fluctuations 13, 14. A complete biophysical theory 
should be able to account for both equilibrium and nonequilibrium steady state (NESS) excitable behaviors 15. In cell physiological terms: There is no need for an active pump to sustain a NP-NJ equilibrium under a common reversal potential $V_{r}$. However, there is a continuous dissipation when there are $\boldsymbol{t w o}$, different reversal potentials $V_{r K}$ and $V_{r N a}$. A difference between two reversal potentials has to be sustained by an active pump such as $\mathrm{Na}^{+} / \mathrm{K}^{+}$-ATPase 12 . See Fig. 1 for an illustration.

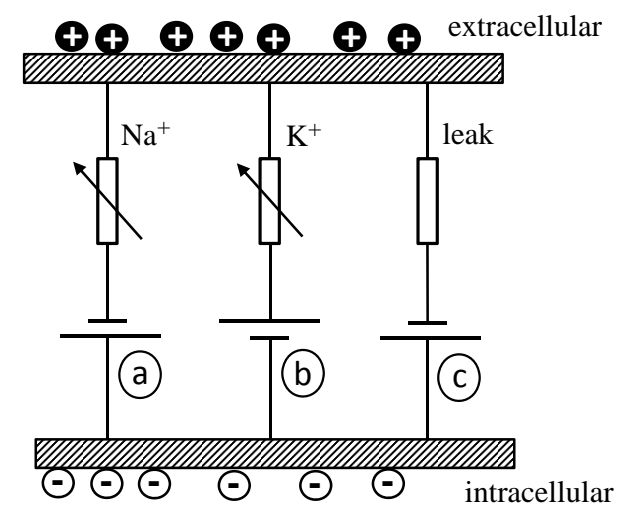

Fig. 1: A standard electrical analogue for HH's electrical membrane with a membrane capacitor (denoted by the two plates) and three types of channels: $\mathrm{Na}^{+}, \mathrm{K}^{+}$, and leak, the first two having variable conductances. The three batteries (a), (b), and (c) represent the reversal potentials for the three respective ions. This electrical diagram shows that if all three batteries are having a same voltage, then the entire system has no current, thus in an equilibirum. However, if any two batteries are having different voltages, then there will be a current going through one of the circuit loops, thus the system is in a nonequilibrium steady state.

This Letter contains two parts. First, it reports a stochastic theory for a passive membrane, as a capacitor with currents leaking through equilibrium fluctuating membrane channel proteins. To simplify the presentation, but without loss of generality, we consider only one type of cation in the electrolyte aqueous solution that surround the membrane. To be consistent with electrochemical NP equilibrium, such a formulation requires a fluctuationdissipation relation, or detailed balance $\left.\begin{array}{lll|l}13 & 15\end{array}\right]$. This yields a previously unknown relation between the voltage-dependent channel conformational transition rates $\lambda_{i j}$ and membrane charge $Q_{m}$ that consists of only mobile ions, excluding the confined charges within membrane proteins. Secondly, with the setup developed, a thermodynamically rigorous nonequilibrium formulation of stochastic HH theory with two types of cations is presented. Entropy production in NESS is studied.

Stochastic theory of fluctuating membrane charges. - There are electrostatic interactions between the charged groups within proteins embedded in a lipid bilayer, channels or not, and the mobile electrical charge density on the membrane, $Q_{m}$ : The conformational states of a channel protein influence the membrane charge via a cross-membrane ionic current and intra-membrane charge/dipole movements, and in return, the membrane charges affect the conformational dynamics of the proteins through their voltage sensors, known collectively as "gating charge" [4 6]. Following NJ's theory, we put this verbal description into a mesoscopic stochastic dynamic model for the electrochemical system 14]:

$$
R_{K_{t}} \frac{d Q_{m}}{d t}+\left(\frac{Q_{m}}{C_{K_{t}}}+\epsilon_{K_{t}}-V_{r}\right)=\sqrt{2 k_{B} T R_{K_{t}}} \xi(t),
$$

in which $\xi(t)$ is a Brownian white noise, and $K_{t}$ is a discrete-state Markov jump process collectively representing conformations of the channel proteins. When $K_{t}=i$, the per 
unit area membrane capacitance and conductance are $C_{i}$ and $R_{i}$ respectively. The reversal voltage $V_{r}$ represents an equilibrium NP potential for the particular conducting ions (see below). Just as the $V_{r}$ is an "effective potential" due to the ionic concentration gradient across the membrane, the $\epsilon_{i}$ is an "effective electrical field potential" induced by the net gating charge(s), or any other confined charge(s) in membrane proteins 17 . In the present work, the membrane with embedded proteins is modelled as two conducting plates with confined charges in between. In principle, even in the absence of $V_{r}$, there is a membrane potential $\epsilon_{i}$; in reality, this effect is miniscule.

The significance of this dynamic description is that it unifies the theories of NJ on membrane electrical fluctuations 13 and $\mathrm{HH}$ on action potentials [1. In fact, denoting membrane potential $V_{m}=Q_{m} / C$, conductance $g=R^{-1}$, and neglecting the fluctuating $\xi(t)$ and small $\epsilon_{K_{t}}$, then Eq. 1 becomes $C d V_{m} / d t+g\left(V_{m}\right)\left(V_{m}-V_{r}\right)=0$, which is the starting point of $\mathrm{HH}$ 1, 8. Several remarks for Eq. 1 are in order.

(i) It is important to point out that the $Q_{m}$ is not the conducting charges across the membrane; rather it is the membrane charge density. See [18 for a derivation of (1). The physically correct way is to start from current formulation as NJ and $\mathrm{HH}$ did, not voltage.

(ii) It is assumed that channel conformational transitions are "instantaneous" without being coupled to a $Q_{m}$ change: $\operatorname{Pr}\left\{K_{t+\Delta t}=j \mid K_{t}=i, Q_{m}(t)=q\right\}=\lambda_{i j}(q) \Delta t+o(\Delta t)$. In chemomechanical systems like motor proteins, this type of model is called Brownian ratchet [19. It excludes the possibility of a tightly coupled simultaneous channel conformational transition and change in membrane $Q_{m}$, the so called power stroke scenario in molecular motor. In mathematical physics, (1) is known as a coupled (or switching) diffusion process 20 .

(iii) The problem of stochastic HH dynamics in terms of $\left(Q_{m}(t), K_{t}\right)$ is different from the channel conformational dynamics with gating charge 21. The latter addresses a single protein dynamics in terms of its structure and energy landscape; it employs Eyring-Kramers type of formalism [4] with which detailed balance is satisfied automatically. The HH theory, however, is a phenomenological model of an electrochemical system that consists of both channels and a membrane immersed in an electrolyte solution. The transition rate $\lambda_{i j}(q)$ represents a macromolecular conformational change in the system's setting. Because of this, the detailed balance, or fluctuation-dissipation relation, has to be enforced as an additional requirement to the stochatic equation (1), as in the earlier work of Einstein and Nyquist [16], and studies on motor proteins [23.

(iv) Indeed, a quantitative isomorphism exists between an electrochemical system and a mechanochemical system such as molecular motors and ligand-receptor dissociation kinetics under an external force [24].

$(v)$ It is assumed that gating charge movements within a channel affect the membrane capacitance as well as changing its permeability for the conducting ions. Therefore, with the $Q_{m}$ remains constant immediately before and after the conformational transition, membrane potential jumps from $\left(Q_{m} / C_{i}+\epsilon_{i}\right)$ to $\left(Q_{m} / C_{j}+\epsilon_{j}\right)$. This is the response to gating sensor movements associated with the conformational transition. The present theory, therefore, provides a quantitative link between molecular physics model of channel gating and the $\mathrm{HH}$ theory. Its validity can and should be experimentally tested.

(vi) The $V_{r}$ accounts for a concentration difference across the membrane that is in equilibrium with a membrane potential. According to NP equation, an ionic concentration gradient across the membrane, say the $\mathrm{K}^{+}$with $c_{1}$ and $c_{2}$, gives rise to an equilibrium membrane potential $V_{r}=\left(k_{B} T / e\right) \ln \left(c_{1} / c_{2}\right)$. In such an equilibrium, the net $\mathrm{K}^{+}$current is zero, with the membrane potential $V_{r}$ driven ionic current exactly balances the concetration gradient driven ionic flux in the opposite direction. However, such an NP equilibrium is conditioned on the absolutely zero counter-ion, e.g., $\mathrm{Cl}^{-}$, passing through the membrane. In reality, if the membrane voltage is not clamped, a slow counter-ion leakage gradually causes both membrane potential and the ion concentration difference to dissipate. The $V_{r}$ in (1) can be treated as a constant only on the time scale of no significant counter-ion leakage. 
(vii) There are several possible lines of experimental and computational tests for this theory. One is an integrative analysis of empirically determined voltage-dependent gating kinetics with computational molecular dynamics in an ionic solution with proper handling of ions and charges. Even though nano-scale Coulomb meter for measuring membrane charge is still in its infancy [6, being able to probe membrane voltage and charges as separated physical quantities will provide a deeper understanding of the complex "capacitance". Finally, beyond single-channel recording, experiments on artificial membrane with two types of ion channels in the presense of two different ionic reversal potentials will open a new vista for nonequilibrium physical biology.

A thermodynamic linkage relation between channel and membrane. - From (1), the probability of $\left(Q_{m}(t), K_{t}\right), f_{Q_{m} K}(q, i, t)$, satisfies the Fokker-Planck-master equation

$$
\begin{aligned}
\frac{\partial f_{Q_{m} K}(q, i, t)}{\partial t}= & \frac{\partial}{\partial q}\left(\frac{1}{R_{i}}\right)\left[k_{B} T \frac{\partial f_{Q_{m} K}(q, i, t)}{\partial q}+\left(\frac{q}{C_{i}}+\epsilon_{i}-V_{r}\right) f_{Q_{m} K}(q, i, t)\right] \\
& +\sum_{j}\left[f_{Q_{m} K}(q, j, t) \lambda_{j i}(q)-f_{Q_{m} K}(q, i, t) \lambda_{i j}(q)\right] .
\end{aligned}
$$

To have an electrochemical equilibrium one has to have both total electrical current and chemical (Fickian) flux for each species being zero simultaneously. Therefore,

$$
\frac{\lambda_{i j}(q)}{\lambda_{j i}(q)}=\frac{f_{Q_{m} K}^{e q}(q, j)}{f_{Q_{m} K}^{e q}(q, i)}=\frac{\lambda_{i j}(0)}{\lambda_{j i}(0)} \exp \left\{-\frac{1}{k_{B} T}\left(\frac{q^{2}}{2 C_{j}}+\epsilon_{j} q-\frac{q^{2}}{2 C_{i}}-\epsilon_{i} q\right)\right\} .
$$

We note that the $V_{r}$ disappeared from (3), as it should be. Eq. 3 is the detailed balance relation for the mixed discrete and continuous variables 22, 23, as illustrated in Fig. 2.

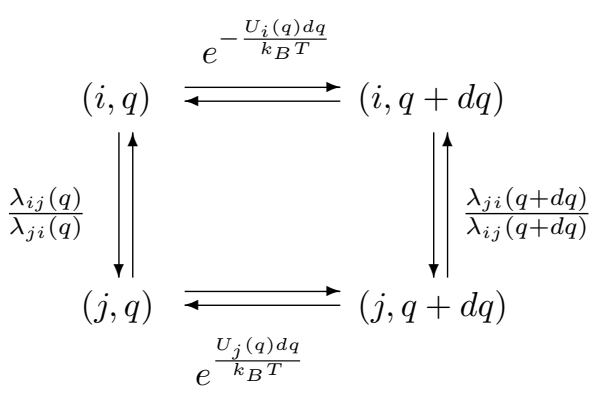

Fig. 2: In the electrochemical theory of a membrane with fluctuating ion channels, detailed balance condition for equilibrium is expressed as a relation between the membrane electrical potential functions $U_{k}(q)=q^{2} /\left(2 C_{k}\right)$ and transition rates $\lambda_{i j}(q)$, as given in Eq. 3. over a kinetic cycle illustrated here.

Voltage-dependent channel conformational transition rates. - Usually, the changes in capacitance due to gating charge moments are much smaller than the contribution from the lipid bilayer. Therefore, we can assume $C_{i}=C_{m}+\delta_{i}$ with $\delta_{i} \ll C_{m}$. In this case, the term on the rhs of $(3)$ becomes $\exp \left\{-\left[q\left(\epsilon_{i}-\epsilon_{j}\right)+q^{2}\left(\delta_{i}-\delta_{j}\right) /\left(2 C_{m}^{2}\right)\right] /\left(k_{B} T\right)\right\}$. Therefore, in terms of the membrane potential $V_{m}=q / C_{m}$ one has

$$
\frac{\lambda_{i j}\left(V_{m}\right)}{\lambda_{j i}\left(V_{m}\right)}=\frac{\lambda_{i j}(0)}{\lambda_{j i}(0)} \exp \left\{-\frac{1}{k_{B} T}\left(\left(\epsilon_{i}-\epsilon_{j}\right) C_{m} V_{m}+\frac{\left(\delta_{i}-\delta_{j}\right) V_{m}^{2}}{2}\right)\right\} .
$$

The $\left(\epsilon_{i}-\epsilon_{j}\right) C_{m}$ term and the $\left(\delta_{i}-\delta_{j}\right)$ term are due to charge movements and changes in dipole moments associated with gating, the conformational change. See Hill and Chen 21 
for more discussions. If $\delta_{i}$ is not much smaller than $C_{m}$, then $V_{m}$ is discontinuous and the "voltage-dependent rate" has to be defined as $\hat{\lambda}_{i j}\left(V_{m}\right) \equiv \lambda_{i j}\left(C_{i} V_{m}\right)$.

The membrane capacitance $C_{m}$ appearing on the rhs of (4) should not be a surprise. "voltage-dependent transition rates" actually depends upon the nature of applied force law, e.g., elastic cantilever or constant force clamping [25]. This means the voltage-dependent transition rates in $\mathrm{HH}$ theory, in principle, are different from those obtained from voltageclamped single-channel recording. However, the $\lambda_{i j}(q)$ are intrinsic properties of a membranechannel system.

Equilibrium electrochemical fluctuations - With the condition given in (3), one has the Boltzmann's law which dictates that in an thermo-electrico-chemical equilibrium, with the stationary probability

$$
\begin{aligned}
f_{Q_{m} K}^{e q}(q, i) & =\mathcal{N}_{1}^{-1} \exp \left(-\frac{\left(q-q_{i}^{*}\right)^{2}}{2 C_{i} k_{B} T}-\frac{\mu_{i}(0)-C_{i}\left(V_{r}-\epsilon_{i}\right)^{2} / 2}{k_{B} T}\right) \\
& =\mathcal{N}_{2}^{-1} \exp \left(-\frac{\mu_{i}(q)-\mu_{1}(q)}{k_{B} T}-\frac{\left(q-C_{1} V_{r}\right)^{2}}{2 C_{1} k_{B} T}\right),
\end{aligned}
$$

in which $q_{i}^{*}=\left(V_{r}-\epsilon_{i}\right) C_{i}$, and

$$
\mu_{i}(q)-\mu_{j}(q)=k_{B} T \ln \left(\frac{\lambda_{i j}(q)}{\lambda_{j i}(q)}\right),
$$

which is independent of $V_{r}$, and

$$
\begin{gathered}
\mathcal{N}_{1}=\sum_{i} \sqrt{2 \pi C_{i} k_{B} T} e^{-\frac{\mu_{i}(0)}{k_{B} T}+\frac{C_{i}\left(V_{r}-\epsilon_{i}\right)^{2}}{2 k_{B} T}}, \\
\mathcal{N}_{2}=\mathcal{N}_{1} e^{\frac{\mu_{1}(0)}{k_{B} T}-\frac{C_{1} V_{r}^{2}}{2 k_{B} T}} .
\end{gathered}
$$

The detailed balance condition (3) guarantees zero entropy production in the stationary stochastic dynamics $[15$.

Nonequilibrium steady state with non-zero entropy production. - For system having two types of cations with different reversal potential $V_{r K}$ and $V_{r N}$, and corresponding Markov channels kinetics $K_{t}$ and $N_{t}$, the membrane dynamics can be represented by an equation parallel to (11). Following HH's theory, we assume that $C_{i} \approx C_{m}$, neglecting $\epsilon$, and denote $V_{m}=Q_{m} / C_{m}$. Then the stochastic HH equation:

$$
C_{m} \frac{d V_{m}}{d t}+g_{K_{t}}\left(V_{m}-V_{r K}\right)+\gamma_{N_{t}}\left(V_{m}-V_{r N}\right)=\sqrt{2 k_{B} T\left(g_{K_{t}}+\gamma_{N_{t}}\right)} \xi(t) .
$$

We note that if $V_{r K}=V_{r N}$, then the two terms on the lhs can be merged into a single term, and the results from the previous section apply. However, if they are not equal, then the stationary process is a NESS. To prove that, we have electrical equilibrium

$$
f_{V_{m} K N}^{n e s s}(v, i, j)=\widetilde{\mathcal{N}}_{i j} \exp \left(-\frac{C_{m}\left(v-v_{i j}^{*}\right)^{2}-C_{m} v_{i j}^{* 2}}{2 k_{B} T}\right),
$$

in which

$$
v_{i j}^{*}=\frac{g_{i} V_{r K}+\gamma_{j} V_{r N}}{g_{i}+\gamma_{j}},
$$

which is related to the Goldman-Hodgkin-Katz diffusion potential 22. Using (11) and if the steady state were detailed balanced between $(i, j)$ and $\left(i^{\prime}, j^{\prime}\right)$ at $v=0$, then one would have

$$
\frac{f_{V_{m} K N}^{n e s s}(v, i, j)}{f_{V_{m} K N}^{n e s s}\left(v, i^{\prime}, j^{\prime}\right)}=\frac{\lambda_{i^{\prime} j^{\prime} \rightarrow i j}(0)}{\lambda_{i j \rightarrow i^{\prime} j^{\prime}}(0)} \exp \left[-\frac{C_{m}\left(v_{i j}^{*}-v_{i^{\prime} j^{\prime}}^{*}\right) v}{k_{B} T}\right] \text {. }
$$


However when $V_{r K} \neq V_{r N}, v_{i j}^{*}$ is a function of $i$ and $j$, and the rhs of 130 can not be equal to $\lambda_{i^{\prime} j^{\prime} \rightarrow i j}(v) / \lambda_{i j \rightarrow i^{\prime} j^{\prime}}(v)$ which has to be independent of $V_{r N}$ and $V_{r K}$. This proves that the stationary process is now a NESS. Note that $\lambda_{i j \rightarrow i^{\prime} j^{\prime}}(v)$ satisfies detailed balanced for each given $v$ 3. The same model, therefore, can account for both equilibrium NP-NJ theory, as well as HH's excitable dynamics in a NESS.

One can in fact obtain the NESS distribution analytically in the case of rapid channel fluctuations 23]:

$$
f_{V_{m} K N}^{n e s s}(v, i, j)=\pi(i, j \mid v) \mathcal{N}^{-1} \exp \left[-\frac{C_{m}}{k_{B} T}\left(\frac{v^{2}}{2}-\int_{0}^{v} \varphi^{*}(y) d y\right)\right],
$$

in which $\mathcal{N}$ is a normalization factor, and

$$
\begin{gathered}
\pi(i, j \mid v) \lambda_{i j \rightarrow i^{\prime} j^{\prime}}(v)=\pi\left(i^{\prime}, j^{\prime} \mid v\right) \lambda_{i^{\prime} j^{\prime} \rightarrow i j}(v), \\
\varphi^{*}(v)=\frac{\sum_{i, j}\left(g_{i} V_{r K}+\gamma_{j} V_{r N}\right) \pi(i, j \mid v)}{\sum_{i, j}\left(g_{i}+\gamma_{j}\right) \pi(i, j \mid v)} .
\end{gathered}
$$

The corresponding NESS entropy production is

$$
e_{p}=\sum_{i, j} \int_{-\infty}^{\infty} \frac{\left(g_{i}+\gamma_{j}\right)\left(\varphi^{*}(v)-v_{i j}^{*}\right)^{2}}{k_{B} T} f_{V_{m} K N}^{n e s s}(v, i, j) d v .
$$

When $V_{r K}=V_{r N}, \varphi^{*}(v)=v_{i j}^{*}$ and $e_{p}=0$.

Summary. - While it is widely accepted that HH's action potential dynamics of an excitable electrical membrane has to be a nonequilibrium phenomenon [2], which is driven by the difference in $\mathrm{Na}^{+}$and $\mathrm{K}^{+}$reversal potentials, there has never been a quantitative statistical thermodynamic theory that adequately account for this intuition. Using the stochastic mathematics of coupled diffusion widely employed in Brownian ratchet theory for molecular motors, we have developed a quantitative model which takes in account both NP-NJ type of charge-chemical equilibrium with fluctuations, and stochastic HH's theory. For the equilibrium part, we discovered a previously unknown relation akin to a detailed balance condition. We show that if there are two ions with unequal reversal potentials, then a NESS arises. In the case of rapid channel fluctuation, we obtained an explicit expression for the NESS entropy production, which becomes zero when the two reversal potentials are equal. This model provides the basis for future investigations of the emergence of organized living phenomenon through nonequilibrium physics.

$$
* * *
$$

We thank F. Bezanilla, I. Goychuk, B. Hille, Bo Li, Wei-Shi Liu, R. Nossal, D. Sigg, P.J. Thomas, Li-Guo Wang, T.B. Woolf and Fu-Xi Zhang for helpful discussions. H.Q. also thanks Profs. Paul De Weer and Luis Reuss for introducing him to membrane biophysics.

\section{REFERENCES}

[1] A. L. Hodgkin and A. F. Huxley, J. Physiol. 177, 500 (1952); W.A. Catterall, I.M. Raman, H.P.C. Robinson, T.J. Sejnowski and O. Paulsen, J Neurosci. 32, 14064 (2012); J. Keener and J. Sneyd, Mathematical Physiology: I: Cellular Physiology, 2nd ed. (Springer, New York, 2008).

[2] B. Hille, Ion Channels of Excitable Membranes, 3rd ed. (Sinauer, MA, 2001); S. G. Schultz, Basic Principles of Membrane Transport (Cambridge Univ. Press, U.K., 1980).

[3] B. Sakmann and E. Neher (eds.) Single-Channel Recording, 2nd ed. (Springer, New York, 1995).

[4] F. Bezanilla and E. Stefani, Annu. Rev. Biophys. Biomol. Struct. 23, 819 (1994); G. Yellen, Q. Rev. Biophys. 31, 239 (1998). 
[5] R. MacKinnon et al. Science, 280, 106 (1998); H. Hong, N. H. Joh, J. U. Bowie and L. K. Tamm, Meth. Enzymol. 455, 213 (2009).

[6] L. Wang, Annu. Rev. Biochem. 81, 615 (2012).

[7] F. R. Fernandez, T. Broicher, A. Truong and J.A. White, J. Neurosci. 31, 3880 (2011); M. Jenkner and P. Fromherz, Phys. Rev. Lett. 79, 4705 (1997); K. Seifert, K. Fendler and E. Bamberg, Biophys. J. 64, 384 (1993).

[8] H. Lecar and R. Nossal, Biophys. J. 11, 1048; 1068 (1971); T.L. Hill and Y.-D. Chen, Biophys. J. 12, 948 (1972).

[9] A.F. Strassberg and L.J. DeFelice, Neural Comput. 5, 843-655 (1993); R. Fox and Y.N. Lu, Phys. Rev. E 49, 3421 (1994); C.C. Chow and J.A. White, Biophys. J. 71, 3013 (1996).

[10] G. Schmid, I. Goychuk and P. Hänggi, Europhys. Lett. 56, 22 (2001); P. Jung and J.W. Shuai, Europhys. Lett., 56, 29-35 (2001).

[11] J. Goldwyn and E. Shea-Brown, PLoS Comp. Biol. 7, 1002247 (2011).

[12] P. De Weer, Annu. Rev. Physiol. 62, 919 (2000); J. C. Skou, Biosci. Rep. 18, 155 (1998); H. Qian and H. E. Autzen, Biophys. J. 103, 1409 (2012).

[13] H. Nyquist, Phys. Rev. 32, 110 (1928); J. B. Johnson, Phys. Rev. 32, 97 (1928).

[14] C. Kittle, Elementary Statistical Physics, (John Wiley \& Sons, New York, 1958); J. Keizer, Statistical Thermodynamics of Nonequilibrium Processes, (Springer-Verlag, New York, 1987); C. Gardiner, Handbook of Stochastic Method for Physics, Chemistry and the Natural Sciences, 3rd ed. (Springer, New York, 2004).

[15] X.-J. Zhang, H. Qian and M. Qian, Phys. Rep. 510, 1 (2012); H. Ge, M. Qian and H. Qian, Phys. Rep. 510, 87 (2012); D.-Q. Jiang, M. Qian and M.-P. Qian, Mathematical Theory of Nonequilibrium Steady States (Springer, New York, 2004).

[16] R. Kubo, Rep. Prog. Phys. 29, 255 (1966); R.H. Fowler and E.A. Milne, Proc. Natl. Acad. Sci. USA 11, 400 (1925); R.C. Tolman, Proc. Natl. Acad. Sci. USA 11, 436 (1925).

[17] In the general discussion on "voltage sensing" of voltage-dependent ion channels, the translocation of a fixed charge, or charges, and movements of dipole moments are two mechanisms. The term $\epsilon_{K_{t}}$ represents, phenomenologically, the former and the $C_{K_{t}}$ represents the latter. We we shall show below, they yield $V_{m}$ and $V_{m}^{2}$ dependence, respectively. See also 21.

[18] Starting with current $I(t)$ and Kirchhoff's law $L \dot{I}+R I+(1 / C) \int I(\tau) d \tau=0$, one considers the charge on the capacitor $Q(t)$ and $L \ddot{Q}+R \dot{Q}+(1 / C) Q=0$. The electrical energy in the system is $E(Q)=L I^{2} / 2+Q^{2} /(2 C)$. For mesoscopic membrane immersed in an electrolyte solution one neglects the inductance $L$, and considers a fluctuating charge term $\sqrt{2 k_{B} T R} \xi(t)$, whose amplitude is determined by NJ theory according to Boltzmann's law $f^{e q}(Q) \propto \exp \left[-E(Q) /\left(k_{B} T\right)\right]$ 13. Incorporating gating current into HH theory was discussed by G. Schmid, I. Goychuk and P. Hänggi, Phys. Biol. 3, 248 (2006).

[19] D. Chowdhury, Phys. Rep. 529, 1 (2013); M. Qian, X.-J. Zhang, R. Wilson and J. Feng, Europhys. Lett. 84, 10014 (2008); H. Qian, J. Phys. Cond. Matt. 17, S3783 (2005); P. Reimann, Phys. Rep. 361, 57 (2002).

[20] M. Qian and F.-X. Zhang, J. Theoret. Prob. 24, 729 (2011); F.-X. Zhang, J. Math. Phys. 46, 063304 (2005); Z.-C. Guo, M. Qian and M.-P. Qian, Acta Math. Appl. Sin. 3, 58 (1987); M. Qian and B. Zhang, Acta Math. Appl. Sin. 1, 168 (1984); M. Qian, B. Zhang and Z.-C. Guo, Chin. Sci. Bull. 29, 1279 (1984); X. Mao and C. Yuan, Stochastic Differential Equations with Markovian Switching (Imp. Col. Press, U.K., 2006); G.G. Yin and C. Zhu, Hybrid Switching Diffusions: Properties and Applications (Springer, New York, 2009).

[21] Continuous diffusion theory for gating kinetics in terms of a "gating charge displacement", was first formulated by T.L. Hill and Y.-D. Chen, Proc. Natl. Acad. Sci. USA 69, 1723 (1972). However, a thorough analysis had not been carried out until much later: P. Laüger, Biophys. J. 53, 877 (1988); D.G. Levitt, Biophys. J. 55, 489 (1989); S. C. Crouzy and F. J. Sigworth, Biophys. J. 64, 68 (1993); D. Sigg, H. Qian and F. Bezanilla, Biophys. J. 76, 782 (1999); D. Sigg and F. Bezanilla, Biophys. J. 84, 3703 (2003).

[22] H. Wang and G. Oster, Nature 396, 279 (1998).

[23] H. Qian, J. Math. Chem. 27, 219 (2000); H. Qian, J. Phys. Chem. B 106, 2065 (2002); H. Qian, P.-Z. Shi and J. Xing, PCCP, 11, 4861 (2009).

[24] I. Tinoco, Jr., Ann. Rev. Biophys. Biomol. Struct. 33, 363 (2004); H. Qian, Protein Sci. 11, 1 (2002).

[25] C.L. Cole, Ph. D. dissertation, Univ. of Washington, Seattle (2011); H. Qian, Bio- 
H. Qian et al.

phys. Chem. 67, 211 (1997). Consider coupled diffusion $\partial u_{1} / \partial t=(\partial / \partial x) D_{1}\left[\partial u_{1} / \partial x\right.$ $\left.+\left(\kappa_{1} x-f_{e x t}(x)\right) /\left(k_{B} T\right) u_{1}\right]-\lambda_{12}(x) u_{1}+\lambda_{21}(x) u_{2}, \partial u_{2} / \partial t=(\partial / \partial x) D_{2}\left[\partial u_{2} / \partial x+\left(\kappa_{1} x-\right.\right.$ $\left.\left.f_{\text {ext }}(x)\right) /\left(k_{B} T\right) u_{2}\right]+\lambda_{12}(x) u_{1}-\lambda_{21}(x) u_{2}$, with $\frac{\lambda_{12}(x)}{\lambda_{21}(x)}=\frac{\lambda_{12}(0)}{\lambda_{21}(0)} \exp \left[-\frac{\left(\kappa_{2}-\kappa_{1}\right) x^{2}}{2 k_{B} T}\right]$. It yields an equilibrium solution $u_{i}^{e q}(x)=\mathcal{N} \lambda_{j i}(0) \exp \left\{-\left[\kappa_{1} x^{2} / 2-\int f_{\text {ext }}(x) d x\right] /\left(k_{B} T\right)\right\}, \mathcal{N}$ being a normalization factor. The external force, conditioned on being in state 1 , is $\bar{f}_{\text {ext }}=$ $\int_{-\infty}^{\infty} f_{e x t}(x) u_{1}^{e q}(x) d x$; and the transition rate $\hat{\lambda}_{12}=\int_{-\infty}^{\infty} \lambda_{12}(x) u_{1}^{e q}(x) d x . \hat{\lambda}_{12}$ as a function of $\bar{f}_{\text {ext }}$ depends upon the force law $f_{\text {ext }}(x)$. Force clamping corresponds to $f_{\text {ext }}(x)=$ const; elastic probe means $f_{\text {ext }}(x)=\kappa(x-z)$ in which $z$ is controlled by an experimenter. 